\title{
Análisis de la violencia de género y su impacto en la mujer estudiante de Policía de la Escuela Carlos Eugenio Restrepo*
}

[Separata]

Héctor Betancur Giraldo ${ }^{* *}$

Recibido: 17 de febrero de 2021

Aprobado: 2 de mayo de 2021

Citar como:

Betancur, h. (2021). Análisis de la Violencia de Género y su impacto en la Mujer Estudiante de Policía de la Escuela Carlos Eugenio Restrepo. Análisis, 53(99).

https://doi.org/10.15332/21459169.6805

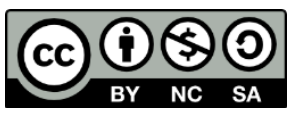

\section{Resumen}

Este artículo permite la comprensión del fenómeno de la violencia de género como problemática social existente en Colombia. A su vez, aborda la violencia de género ejercida contra la mujer estudiante de policía de la Escuela de Policía Carlos Eugenio Restrepo. En este se indagó sobe las percepciones, conocimientos, posiciones y tipos de violencia de género que, en opinión de las estudiantes, se presentaron en la descripción de algunos hechos dados en el proceso formativo. Esto, permitió el acercamiento con la presencia del fenómeno en los hallazgos obtenidos,

\footnotetext{
* Artículo de Investigación.

** Ph. D (c) en Filosofía y Letras Universidad de Alicante. Magíster en Educación U. De Medellín, Especialista en Educación, Cultura y Ciencia Política. Licenciado en Filosofía, Sociólogo. Director de Investigación e Innovación Unisabaneta. Líder del Grupo "Policía y Sociedad". ESCER. Correo electrónico: tobybetan5@gmail.com; ORCID: https://orcid.org/0000-0002-5252-8654
} 
formulándose sugerencias sobre políticas institucionales para enfrentarlo en la institución policial. Asimismo, fue un trabajo pensado en la construcción de relaciones de equidad y respeto por los Derechos Humanos de la mujer policía. Finalmente, se evidenció que las estudiantes reconocieron la existencia del fenómeno en la institución, su nivel de importancia, ámbitos más representativos, la identificación de los principales actores, manifestaciones y acciones violentas, los actos de discriminación y el conocimiento de las normas que las protegen en la institución policial y en la sociedad.

Palabras clave: estudiante, género, mujer, policía, violencia.

\section{Analysis of Gender-based Violence and its Impact on Female Police Students at the Escuela Carlos Eugenio Restrepo}

\section{Abstract}

This article allows the understanding of the phenomenon of genderbased violence as a social problem in Colombia. At the same time, it addresses gender-based violence against female police students at the police school Escuela de Policia Carlos Eugenio Restrepo. It inquired about perceptions, knowledge, positions and types of gender-based violence that, in the students' opinion, were presented in the description of some facts given in the training process. This allowed the approach with the presence of the phenomenon in the findings obtained, presenting suggestions on institutional policies to deal with it in the police institution. Likewise, it was a work designed to build relationships of equity and respect for the human rights of policewoman. Finally, it was observed that the students recognized the existence of the phenomenon in the institution, its level of importance, the most representative areas, identification of the main actors, violent demonstrations and actions, acts of discrimination and knowledge of the rules that protect them in the police institution and in society.

Keywords: student, gender, woman, police, violence. 


\section{Análise da violência de gênero e seu impacto nas mulheres que estudam no Colégio Carlos Eugenio Restrepo para serem policiais}

\section{Resumo}

Este artigo permite compreender o fenômeno da violência de gênero como um problema social existente na Colômbia. Ao mesmo tempo, aborda a violência de gênero contra as mulheres que estudam na Escola de Polícia Carlos Eugenio Restrepo. Neste estudo, foram investigadas as percepções, conhecimentos, posicionamentos e tipos de violência de gênero que, na opinião das estudantes, foram apresentados na descrição de alguns fatos dados no processo de formação, o que permitiu uma aproximação com a presença do fenômeno nos resultados obtidos, formulando sugestões de políticas institucionais para enfrentá-lo na instituição policial. Da mesma forma, foi um trabalho destinado a construir relações de equidade e respeito aos Direitos Humanos da mulher policial. Por fim, constatou-se que as estudantes reconheceram a existência do fenômeno na instituição, seu nível de importância, as áreas mais representativas, a identificação dos principais atores, manifestações e ações violentas, atos de discriminação e conhecimento das normas que as protegem na instituição policial e na sociedade.

Palavras-chave: estudante, gênero, mulher, polícia, violência.

El problema de la mujer siempre ha sido un problema de hombres.

Simone de Beauvoir (1908-1986)

\section{Introducción}

Esta investigación puso en escena la violencia de género, específicamente contra las mujeres estudiantes de policía de la Escuela Carlos Eugenio Restrepo; en esta se pudo determinar el tipo de violencia por medio de las percepciones, conocimientos y posiciones. De esta forma, la investigación 
permitió la realización de un diagnóstico y análisis de las manifestaciones de esta problemática en el proceso de formación policial, con el fin de identificar el fenómeno y sus características en este contexto; por tanto, se dio un acercamiento sobre la forma como se manifiesta en la institución a partir de los resultados, y desde un enfoque exploratorio se planteó la generación de recomendaciones, estrategias y políticas para lograr un impacto en el manejo y tratamiento del fenómeno en el interior de la institución policial.

Si bien es cierto que la institución policial promueve discursos incluyentes y de respeto por los derechos humanos, también es cierto que los procesos que se desarrollan en sus dinámicas estructurales revelan una transformación lenta, al estar sustentados en la conformación de órdenes jerárquicos establecidos, los cuales pueden generar, reproducir y naturalizar culturalmente la violencia de género contra las mujeres. Estas formas de violencias estructurales prevalecen a través de algunos mecanismos discriminatorios y la inequidad de género en una organización androcéntrica, de tradición masculina y militarista en la que se fundamentan las fuerzas armadas en Colombia.

Por tanto, fue fundamental indagar sobre la percepción, conocimientos y posiciones en cuanto a la problemática que experimentan las mujeres estudiantes de policía, que estuvieron enmarcadas en las violencias que se desprenden de los órdenes jerárquicos, entre ellas las de género, donde los mecanismos de subordinación y ejercicio del poder son hegemónicos y se basan en la premisa del cumplimiento de las órdenes en la Policía Nacional por encima de las relaciones humanas y de sus integrantes. Esta exploración del fenómeno estudiado en el centro de formación policial permitió identificar estos tipos de violencia, entre los que se encontraron la física, la sexual, la psicológica y la económica, de los que fueron víctimas las estudiantes. 
De allí, se pudieron generar recomendaciones, sugerir estrategias de prevención y fortalecimiento de las políticas para la prevención y protección de los derechos, y garantías de equidad e igualdad de género hacia la mujer en la institución policial. Para finalizar, dentro de las intencionalidades se sugirió, a partir de los resultados de la investigación, establecer escenarios de discusión y análisis para la prevención, sensibilización e implementación de políticas institucionales para la mujer policía, resaltando la importancia de esta y de su reconocimiento por el papel que cumple en la sociedad como engranaje y baluarte estratégico para el desarrollo, evolución y progreso de la institución y del país.

\section{Metodología}

Se trató de una investigación mixta, exploratoria, de campo y documental, para determinar por medio de un análisis profundo la existencia de violencia de género en el ámbito de la formación policial. Se desarrolló en el año 2019-2020 en la Escuela de Policía Carlos Eugenio, donde estaban en proceso de formación un total de 690 mujeres estudiantes de policía, allí se seleccionó una muestra del $10 \%$, representado en 69 mujeres para la aplicación de una encuesta y dos mujeres más para entrevista y participación en el proceso investigativo. Esto se llevó a cabo con la finalidad de profundizar en el estudio y conocimiento de las percepciones, posiciones y tipos de violencia de género que poseían las mujeres estudiantes de policía. Hernández, et ál., (2014) afirman:

Los métodos mixtos representan un conjunto de procesos sistemáticos, empíricos y críticos de investigación e implican la recolección y el análisis de datos cuantitativos y cualitativos, así como su integración y discusión conjunta, para realizar inferencia producto de toda la información recabada (metainferencias) y lograr un mayor entendimiento del fenómeno bajo estudio. (p. 534) 
Este tipo de investigación proporciona alternativas para el análisis y desarrollo del ejercicio académico con cierto nivel de profundidad, logrando establecerse la integración sistemática de los métodos cuantitativo y cualitativo en un solo estudio. Es así como: "La investigación exploratoria tiene como objetivo examinar o explorar un problema de investigación poco estudiado o que no ha sido analizado antes" (Cazau, citado en Abreu, 2012, p.191). Hay un acercamiento con las características de la problemática, facilitando los mecanismos explicativos que dieron fundamento a la construcción teórica y conceptual de la investigación y como soporte de la hipótesis inicial para conducir el estudio a una mayor profundidad metodológica.

Esto "Ayuda a garantizar que un estudio más riguroso y concluyente en el futuro se iniciará con una comprensión adecuada de la naturaleza del problema de investigación" (Zikmund, citado en Abreu, 2012, p. 192). De esta forma, conduce a dimensionar la importancia de la investigación exploratoria, ya que permite generar insumos para el abordaje teórico y metodológico de la problemática estudiada en el interior del centro de formación policial. En otro aspecto, se lograron definir criterios de comprensión de los resultados, la integración de instrumentos tipo encuestas y entrevistas, y se adhiere una rigurosa revisión de las normas y documentos para el soporte teórico-conceptual para la investigación. Por otra parte, la investigación tuvo un soporte documental que, al ser de carácter exploratorio, incorporó elementos como el análisis riguroso de las fuentes primarias, la selección de libros, artículos de investigación, publicaciones en revistas indexadas, tesis, documentos, conferencias y seminarios, entre otros textos de rigurosidad teórica, conceptual, académica y científica. 


\section{Complejidad de la violencia de género y el feminismo}

Teniendo en cuenta que la violencia de género es una temática analizada en el campo de las ciencias sociales y humanas, entre otras áreas de saber, se puede abordar desde diferentes perspectivas de profundización; por esto, su proceso de configuración tiene una dinámica compleja, toda vez que las áreas de conocimiento que pretenden abordarla están en estrecha relación con casi la totalidad de las relaciones humanas de los individuos en sociedad. De ahí que, se puede iniciar con la Escuela de Estudios de Género de la Universidad Nacional de Colombia, que define este tipo de estudios de la siguiente manera:

Son aquellos cuyo objeto central es el análisis de las interacciones entre hombres y mujeres, el acceso de unos y otros a los bienes y servicios, los cambios culturales, la formación de las identidades, y su énfasis es la comparación y las diferencias entre los sexos. (Puyana, 2004, p. 119)

De ello puede inferirse que los estudios de género permiten establecer un análisis de la descripción de los antecedentes que se desprenden de las relaciones sociales entre los hombres y las mujeres, lo que genera la reflexión acerca de los escenarios de equidad o inequidad que están inmersos en las relaciones mediadas por las diferencias sexuales, el machismo y el patriarcado en la sociedad y en la interacción social de los dos géneros. En otros términos, es "aquella violencia basada en las relaciones y definiciones de género dominantes en una sociedad dada" (Espinar y Mateo, 2007, p.189). Esta situación, además, se articula a argumentos sobre las transformaciones socioculturales y sobre los elementos implícitos en la complejidad del fenómeno social. Estrada (1997):

El feminismo se ha debatido históricamente en torno al complejo problema de la igualdad y la diferencia, apuntando a resolver la aparente 
paradoja en términos de afirmar la igualdad-equidad, ético-política y la diferencia en las prácticas y condiciones sociales de la existencia personal y colectiva. (p. 3)

De acuerdo con esto, se realiza un análisis del manejo de la información sobre las consecuencias humanitarias y la violencia de género existente en Colombia y, en especial, en Medellín; esto, por medio de un estudio analítico propositivo, con el fin de generar algunas iniciativas de información orientados a "dar claves para su potenciación y orientar ejercicios futuros a partir de la solución a los obstáculos presentes en los procesos actuales" (Valencia, 2014, p. 49). Entre las conclusiones, se encuentra que "En términos generales, todos los sistemas de información analizados utilizan un modelo similar para la recolección, procesamiento y generación de informes que comprenden elementos tanto de estructura como de proceso, claramente identificados" (Valencia, 2014, p. 65).

En el mismo contexto, en cuanto a las construcciones sociales sobre la violencia de género, fue realizada una investigación sobre la identificación de atributos y estereotipos de la masculinidad y la feminidad asociados al ciclo de la problemática en mujeres españolas, además de factores relacionados con la violencia doméstica contra la mujer en una población de 1200 sujetos de ambos sexos, mayores de 18 años y de diferentes partes de España. Delgado, et ál., (2012) dicen:

Considerando que las construcciones sociales respecto a las características y roles sexuales muestran una asociación con las interacciones de género en el ciclo de la violencia doméstica, cabe mencionar que en relación con la autovaloración de atributos de feminidad y masculinidad. (pp. 774-775).

Así es dable llegar a la conclusión de que también se encuentran resultados en este trabajo respecto a los índices de violencia doméstica, donde "existe 
un $5.9 \%$ de sujetos que han sufrido violencia de género, así como un 8.5\% que lo sufren en la actualidad” (p. 773). Así, se finaliza con algunos de los hallazgos que destacan las características estereotípicas del maltratador, siendo asociadas al cobarde, agresivo, violento, celoso, posesivo, trastornado, dominante, entre otras.

\section{La violencia de género contra la mujer, un fenómeno social en la Policía Nacional}

La Organización Mundial de la Salud OMS (2013), considera la violencia contra la mujer, también llamada violencia de género como un problema de salud pública y una violación de sus derechos humanos. A nivel mundial, las cifras revelan que el $35 \%$ de las mujeres han sufrido algún tipo de violencia por parte de su pareja o de terceros. Esto constituye en un fenómeno que ha acaparado la atención de algunos investigadores en los últimos años a nivel mundial. Así, las Naciones Unidas definen la problemática en el artículo 1 de la Declaración sobre la eliminación de la violencia contra la mujer (1993) como:

Todo acto de violencia, basado en la pertenencia al sexo femenino, que tenga o pueda tener como resultado un daño o sufrimiento físico, sexual o psicológico para la mujer, así como la amenaza de tales actos, la coacción o la privación arbitraria de la libertad. (ONU, 1993)

Del mismo modo, en la Cuarta Conferencia Mundial de las Naciones Unidas sobre la Mujer se analizaron e investigaron varios aspectos relacionados con las formas de violencia machista contra las mujeres en el mundo. Así, Pérez del Río (2006) afirma: "La violencia contra la mujer constituye una violación de sus derechos humanos y contra sus libertades fundamentales y es una de las más graves consecuencias de las desigualdades económicas, sociales, políticas y culturales que existen entre hombres y mujeres" (p. 185). En efecto, en Colombia los esfuerzos de la 
investigación sobre la violencia contra las mujeres han estado dirigidos mayormente a conocer las características de "las víctimas y en la identificación de las consecuencias de los episodios violentos, esto con el fin de propiciar la denuncia para que la violencia haga tránsito al ámbito de lo público y el Estado dé respuesta a este fenómeno" (Gómez, Murad y Calderón, 2013, p. 11). De esta forma, pocos estudios hacen referencia al fenómeno en instituciones castrenses o policiales en el país.

Asimismo, la violencia de género es un fenómeno que no ha sido explorado, analizado, ni estudiado a profundidad en el interior de una fuerza como la Policía Nacional de Colombia. No obstante, es una fuerza en la que tienen proyecto de vida algunas mujeres y desde sus inicios no se tuvo una proyección desde una perspectiva de género, ni de violencia basada en género, "ya que la participación de esta se vio impulsada por Carola Correa de Rojas Pinilla y su hija María Eugenia Rojas de Moreno" (Policía Nacional de Colombia, 201ob, p. 61). Su inclusión tuvo una visión humanitaria y estigmatizada en función de los roles que en ese momento debían desempeñar, en ese sentido, la incorporación de la mujer en la institución se da desde 1953, como estudiante o como profesional, ya que al ser una institución de tradición androcéntrica, militarista y castrense tiende a invisibilizar la presencia de la mujer.

Sin embargo, a partir de la década de los años noventa, producto de la guerra contra el narcotráfico y el recrudecimiento del conflicto bélico colombiano, la institución empezó a incorporar a la mujer en este tipo de escenarios laborales y a integrar las unidades operativas de la institución policial. En la Constitución Política de Colombia en su artículo 13 se declara.

Todas las personas nacen libres e iguales ante la ley, recibirán la misma protección y trato de las autoridades y gozarán de los mismos derechos, libertades y oportunidades sin ninguna discriminación por razones de 
sexo, raza, origen nacional o familiar, lengua, religión, opinión política o filosófica. (Const., 1991, art. 13)

Por esta condición, se puede concluir con que el desempeño de la mujer en la policía debe estar en igualdad de condiciones a la de los hombres, en cuanto a la equidad y el respeto de sus derechos como estudiante de policía y en su labor como profesional de policía.

\section{La mujer en las fuerzas armadas y de policía}

El aporte actual de los estudios de género al proceso de democratización, sobre todo en el campo institucional, es la incorporación del tema de la equidad de género, el cual viene cobrando cada vez mayor legitimidad y presentando grandes avances en términos de normativas y acceso pleno de las mujeres en campos institucionales que anteriormente les habían sido negados. Así, se analizan los reductos regionales asociados al androcentrismo como el ámbito de la Defensa en Argentina, Colombia, Chile, Ecuador y Uruguay.

[A su vez] este fenómeno de empoderamiento se extendió también al acceso de las presidencias nacionales, como es el caso de Michelle Bachelet [...] en Chile y Cristina Fernández de Kirchner [...] en Argentina, convirtiéndose esta última en la primera mujer Comandante en Jefe de las Fuerzas Armadas en la región en conformidad con la Constitución nacional. (Lucero, 2008, p. 2)

Estas formas de discriminación y menosprecio de las capacidades y del papel de la mujer en la sociedad eran posibles, pues se consideraba que las instituciones del orden armado en el país tenían una condición de exclusividad masculina, orientados desde una doctrina para la guerra y donde se supone las mujeres no poseían ni las condiciones, ni las capacidades de hacer parte de una confrontación armada. Por tanto, la incorporación de la mujer se da en la década de los setenta en las fuerzas 
armadas chilenas y en las paraguayas y se fue reproduciendo de manera paulatina en la región (Lucero, 2008).

En general, hacia la década de los ochenta en Latinoamérica su incorporación tuvo varios ciclos; al principio "fue un tanto periférica y estereotipada, para luego avanzar con la incorporación formal abriendo las armas a las mujeres. Esto resultó el primer paso de remoción de obstáculos normativos y formales desde lo institucional" (Derdoy, 2013, p. 82). Posteriormente, se empiezan a adoptar medidas para garantizar políticas públicas de equidad de género dentro de las instituciones que garanticen el justo disfrute de los derechos por parte de la mujer militar y la mujer policía y su "incorporación plena", es decir, "la asunción de funciones y roles jerárquicos y de mando dentro de la estructura militar, así como el ejercicio pleno de las prerrogativas profesionales" (Bobea, 2008, p. 64); estas son condiciones que le permiten a las mujeres contar con el rango adecuado y con la garantía de dirección y mando. "El proceso fue lento y complejo, pues dichos espacios institucionales habían sido dominados por fuertes regímenes de tradición masculina y un recio patriarcalismo representado en estructuras verticales que los constituía como espacios herméticos" (Ruiz, 2013, p. 2). Además, "El debate internacional sobre la conveniencia de la incorporación de las mujeres a las Fuerzas Armadas ha dado paso a la discusión sobre su participación en roles de combate directo" (Villalobos, 2010, p. 8).

También, están las denominadas "feministas antimilitaristas" que coinciden en que el enfoque feminista "se opone a lo militar por su uso de la diplomacia violenta, por demás asociado al virulento masculinismo de la cultura militar" (Bobea, 2008, p. 68). En oposición, se encuentran las y los defensores del "feminismo igualitario militarista", quienes creen en la participación de la mujer en tareas militares y su incorporación plena en las instituciones castrenses. Así pues, "la incorporación de mujeres a las 
fuerzas armadas no solo ha ayudado a ampliar sus roles y oportunidades profesionales, sino que también ha contribuido a la profundización y consolidación del sujeto ciudadano femenino” (Bobea, p. 68).

En ese mismo sentido, el proceso de feminización de las fuerzas armadas y policiales se consolida en función de la aceptación de la Resolución 47/2 de las Naciones Unidas sobre adopción de la perspectiva de género en las políticas y programas de la ONU, obliga a las fuerzas armadas a asumir la perspectiva de género y los derechos de las mujeres como auténticos derechos humanos de dignidad, pero es claro que es necesario "evitar el fetichismo normativo y el simple avance formal, [para esto] es necesaria la constante revisión de prácticas institucionales que muchas de ellas continúan siendo excluyentes y consolidando la identidad militar como únicamente masculina y varonil” (Derdoy, 2013, p.83).

Así, el paso a seguir en las fuerzas militares es el de preservar el orden y transformarse en "fuerzas armadas posmodernas", es decir, configurarse y transformar sus estructuras tradicionales en "un ejército de pautas flexibles y reclutamiento voluntario [...] formador de recursos humanos profesionales, con roles diversos y de mayor inclusión social" (Moskos, et ál., citados en Lucero, 2008, p. 11). Cabe concluir que estas características permiten a las fuerzas militares y de policía ser garantes del respeto de los derechos humanos, no solo de los ciudadanos, sino en igualdad para sus integrantes, con criterios de equidad y participación en su estructura organizacional y en la prestación efectiva del servicio ante la comunidad en el país.

\section{Configuración de la violencia de género en la sociedad}

Se debe comprender que el fenómeno de la violencia de género se sitúa dentro de la violencia estructural, con una representación en el orden de la violencia física, la cual está arraigada en la cultura y en las prácticas de una 
sociedad como la colombiana, ya que esta se configura por un orden patriarcal, androcéntrico y machista. De ahí que el otro (mujer) haya sido pensado como un sujeto dominado y sobre quien es legítimo ejercer múltiples formas de supresión de su voluntad, sus derechos y hasta, en algunas ocasiones, arrebatarle la misma vida. Estas son manifestaciones de violencia configuradas en un fenómeno altamente pernicioso, donde la sociedad que las reproduce las justifica y, en ocasiones, las invisibiliza, haciendo ver como natural dichas conductas e incorporándolas a las construcciones culturales e idearios sociales. "La deconstrucción, que es una forma de crítica postestructural, cuestiona que los primeros principios propuestos, en los que supuestamente se fundamentan los programas y significados estructuralistas, lleguen a trascender nuestros textos y prácticas discursivas" (Cherryholmes, 1999, p. 30).

Así que, su objetividad radica en poder dejar de lado los tintes dogmáticos e ideológicos de influencia machista y patriarcal que han sido formas de reproducción del fenómeno. Sin embargo, se resalta que el postestructuralismo posee un alto nivel de importancia, al momento de analizar que Venegas (2010) resalta las posturas feministas, cuando afirma "Frente a ello, Butler opta por el postestructuralismo para poner el énfasis en la resistencia y en el cambio social" (Venegas, 2010, p. 150). Por tanto, se invita a la reflexión y transformación sobre las formas de violencia contra la mujer, que se han visto influenciadas por los dispositivos que generan, reproducen y naturalizan la cultura violenta sobre estas; esto, en medio de los modelos machistas hegemónicos que aún se dan en el país.

Se hace énfasis en algunos escenarios poco explorados como el ámbito castrense y policial; por tanto urge la necesidad de escudriñar objetivamente en aportes teóricos como los de Foucault en la descripción sobre el poder y el control, a través de la sexualidad, que generan algunos fundamentos sobre el estudio del fenómeno; por tanto, se puede observar 
conceptualmente que: "Más bien se ha construido un artefacto para producir discursos sobre el sexo, siempre más discursos, susceptibles de funcionar y de surtir efecto en su economía misma" (Foucault, 1976, p. 16). Se debe enfatizar en que: "Tal técnica quizá habría quedado ligada al destino de la espiritualidad cristiana o a la economía de los placeres individuales si no hubiese sido apoyada y reimpulsada por otros mecanismos” (p. 16).

En efecto, Foucault plantea que el control sobre el sexo posee una funcionalidad al ser utilizado como artefacto de control, ya que posee efectos directos hasta en la economía; cuando establece las acciones de sometimiento sobre el cuerpo femenino y, en especial, sobre el sexo y el género, que están dirigidas al control que ejercen los dispositivos de poder en la sociedad machista, logrando dimensionar racionalmente la efectividad que posee el sexo sobre la voluntad del poder, el cual siempre ha sido un dispositivo al servicio del machismo como medio para dominar y ejecutar la acción hegemónica.

De ahí que históricamente se legitimaban acciones instrumentales sobre su género y condición, por lo que describe Foucault (1976) como la "Histerización del cuerpo de la mujer: triple proceso según el cual el cuerpo de la mujer fue analizado - calificado y descalificado- como cuerpo integralmente saturado de sexualidad" (p. 62). Esto conduce, a comprender la intencionalidad sobre el cuerpo de la mujer como objeto de control y dominio, al cosificarla e instrumentalizarla históricamente. De ahí que: "Género y cuerpo son indisociables el uno del otro, constituyéndose mutuamente como materialidades discursivas, creando un yo con un género incorporado (embodied) que se alimenta tanto de la propia subjetividad (generalizada) como de las interacciones" (Enguix y González, 2018, p. 3). 
De esta forma, la hegemonía masculina se evidencia en su accionar; por tanto: "Foucault desarrolla un complejo y amplio trabajo que permite pensar de otra manera: subraya el carácter productivo del poder e insiste en el vínculo saber-poder y en la economía política de la verdad" (Amigot y Pujal, 2009, p. 121). De todo esto resulta que la utilización del cuerpo a partir de la explotación ha permitido infringir acciones para su dominio desde la sexualidad y el género:

El dispositivo de alianza, con los mecanismos coercitivos que lo aseguran, con el saber que exige, a menudo complejo, perdió importancia a medida que los procesos económicos y las estructuras políticas dejaron de hallar en él un instrumento adecuado o un soporte suficiente.

(Foucault, 1976, p. 63)

\begin{abstract}
Ahora bien, el fenómeno ha sido abordado de forma tradicional desde el aparato ideológico y hegemónico que regula la sociedad, procurando ocultar la gravedad de la problemática, ya que es recurrente en escenarios tradicionales la familia, la escuela, la religión, la economía, lo artístico y cultural, en medios de comunicación, entre otros, y que facilitan el estudio del fenómeno y permiten visibilizar la realidad de la violencia de género contra la mujer en Colombia.
\end{abstract}

Hay también toda una campaña de prensa en los periódicos obreros: sobre el tema de que el gobierno favorece el trabajo en las prisiones para hacer que bajen los salarios "libres"; sobre el tema de que los inconvenientes de estos talleres de prisión son todavía mayores para las mujeres, a las cuales quitan su trabajo, empujan a la prostitución, y por lo tanto a la prisión, donde esas mismas mujeres, que no podían trabajar ya cuando eran libres, vienen entonces a hacer la competencia a las que aún tienen trabajo. (Foucault, 2002, p. 221)

De la misma forma, Michel Foucault evidencia teóricamente en su obra Vigilar y castigar, por medio de episodios donde las mujeres empezaron a 
generar acciones en contra de las formas de dominio y sometimiento tradicional a través de apartados históricos que se manifiestan en contra de su libertad y con un rechazo ante la realidad vivida. Esto actúa como reacción ante las instituciones sociales de control y poder que reproducían la violencia machista y misógina y que fueron documentadas en textos como testimonio y patrimonio histórico para exaltar la lucha de las mujeres por sus derechos.
Abajo el cura, decían; abajo ese hombre execrable; se hubiera debido hacer justicia con él. Sin la energía y la firmeza de la guardia municipal, hubieran podido cometerse graves desórdenes. En Vaugirard, eran las mujeres las más furiosas. Gritaban: iAbajo el mal sacerdote! iAbajo el monstruo Delacollonge! Los comisarios de policía de Montrouge, de Vaugirard y varios alcaldes y tenientes de alcalde acudieron, con el fajín desplegado, para hacer respetar la sentencia de la justicia. (Foucault, 2002, p. 238)

En ese sentido, se expone un panorama de acciones que muestran un deseo de la mujer de hacer justicia y ser reconocidas en una sociedad machista que las limita y cosifica desde las estructuras hegemónicas, entre las que se resaltan las encargadas de regular la conducta humana. Entonces, emerge la importancia de argumentar teórica y conceptualmente la reflexión sobre la urgencia por lograr procesos de transformación en la sociedad colombiana mediante procesos estructurales de cambio.

Y siempre he visto en la dominación masculina, y en la manera como se ha impuesto y soportado, el mejor ejemplo de aquella sumisión paradójica, consecuencia de lo que llamo la violencia simbólica, violencia amortiguada, insensible, e invisible para sus propias víctimas, que se ejerce esencialmente a través de los caminos puramente simbólicos de la comunicación y del conocimiento o, más exactamente, del 
desconocimiento, del reconocimiento o, en último término, del sentimiento. (Bourdieu, 1988, pp. 11-12)

De este modo, se puede comprender que, tanto para Foucault como para Bourdieu, las acciones de dominación masculina, androcéntrica y machista representan un afán racional por mantener la hegemonía, el sometimiento y el control sobre la mujer por los mecanismos incluidos en la violencia simbólica. Aun así, en la obra de Foucault La tecnología del yo, se da un análisis profundo sobre los medios, procedimientos y objetivos que construyen las relaciones de poder en la comprensión de las relaciones entre dominador-dominado, sobre las que la sociedad colombiana se ha estructurado en medio de la instauración de las estrategias machistas de dominio sobre la mujer, siendo mecanismos racionales y actuando como violencia de género contra la mujer.

Asimismo, en la construcción teórico-simbólica de la investigación se logra establecer que este fenómeno contra la mujer, más allá de ser una acción instrumental, al ser usada de forma racional por la hegemonía e imponer sus formas de dominio sobre la mujer desde los discursos de poder, representa las acciones permanentes de imposición del androcentrismo y el machismo; por esta razón, queda para las mujeres una alternativa de reflexión:

En consecuencia, los que resisten o se rebelan contra una forma de poder no pueden satisfacerse con denunciar la violencia o criticar una institución. No basta con denunciar la razón en general. Lo que hace falta volver a poner en tela de juicio es la forma de racionalidad existente.

(Foucault, 2008, p. 139)

En conclusión, se establece que la lucha de la mujer debe ser permanente y con mayor ahínco, ya que no basta con establecer escenarios de 
concienciación, sino que el trabajo debe ser insistente contra el orden establecido por la hegemonía machista.

\section{Relaciones de Igualdad, equidad de género y derechos humanos}

La violencia de género se sustenta en la desigualdad de las mujeres en las estructuras sociales dominadas por el patriarcado y la inequidad presentada en la asignación de roles; sin embargo, es pertinente definir que "La categoría de género sigue usándose, en la actualidad, muy frecuentemente, aunque no de forma específica, sino como un término tipo cajón de sastre, ni como un término paraguas que se construye mediante lugares comunes o nociones confusas" (Amigot y Pujal, 2009, p.118). Estas condiciones simbólicas se usan para evitar las sistemáticas "violaciones a los derechos humanos que eran sentidas mayoritariamente por mujeres y que, por lo tanto, no eran visibles con la perspectiva androcéntrica" (Facio, 2003, p. 8). En ese sentido, la lucha de la mujer siempre ha tratado de reivindicar sus derechos en sociedad; por tanto, ha sido una disputa permanente por lograr la equidad de género. "Estos cambios interpelan a las organizaciones sociales entre las que se encuentran las Fuerzas Armadas, quienes, al igual que otras instituciones, iniciaron un proceso de adaptación contextual” (Zubieta, et ál., 2011, p. 103).

Cabe señalar que estos procesos han demandado transformaciones sociales en todas las instituciones del Estado, incluyendo a sus fuerzas de seguridad, además de "otorgar un trato no discriminatorio, pero el principio de igualdad requiere que a veces se les dé un trato idéntico a hombres y mujeres, y a veces uno distinto" (Castrillón y Von Chrismar, 2013, p. 94). Esto se logra, entendiendo la articulación que debe existir entre el principio de igualdad de género y el de no discriminación como relación desde el principio de equidad, el cual "sería parte sustantiva para el logro de la igualdad de género, en el sentido de tener como propósito el 
contribuir al logro de la misma por encima de las diferencias que pudiesen existir" (García citado en Castrillón y Von Chrismar, 2013, p. 94).

En esta misma línea se afirma que "la equidad se hace posible cuando el trato que se les da a las personas está basado en la consideración justa de las necesidades e intereses impuestos por la diferencia" (Castrillón y von Chrismar, p. 94). En los países latinoamericanos, la democratización es una tarea inconclusa en todos los espacios de acción social, ya sean públicos o privados donde se manifiestan violaciones a los derechos humanos y se presentan situaciones en las cuales claramente se representa el otro como un sujeto privado de derechos, donde las víctimas más recurrentes son las mujeres, esto porque las instituciones sufren procesos de democratización.

Dicho proceso de democratización inició en muchos países del subcontinente hacia la década de los ochenta; en esta la sociedad organizada, activistas en pro de la defensa de los derechos de las minorías, grupos vulnerables, entre otros, se movilizaron para recuperar los espacios democráticos perdidos en décadas anteriores frente al accionar de sectores autoritarios de la sociedad, los cuales habían cooptado el Estado a través de las múltiples dictaduras y de violencias contra los sectores progresistas de la sociedad. (Rebolledo, 2001)

A través de este debate intelectual, la democracia fue entendida como un sistema que debía garantizar el respeto a los derechos humanos en todos los aspectos de la vida y garantizar la divergencia política y la participación en la vida pública. En estas luchas por el reconocimiento, las y los activistas en defensa de los derechos de las mujeres y de género tuvieron un papel muy importante, marcando la pauta de acción a través de una militancia comprometida con la idea de transformación social, la igualdad y el respeto a las diferencias. 


\section{Diferenciación entre género, mujer y patriarcado}

En materia de análisis sobre la construcción conceptual de la definición de género, se reviste de un alto nivel de complejidad; por tanto, el “'género' es un concepto que, si bien existe desde hace cientos de años, en la década de los setenta empezó a ser utilizado en las ciencias sociales como categoría con una acepción específica" (Butler, 1996, p. 97). También es cierto que se plantean al respecto una gran cantidad de teorías que tratan de determinar las diferencias que marcan la naturaleza humana tanto de hombres como de mujeres; por tanto, son particularidades que no se desarrollan solo en el contexto de la academia y la investigación, sino que se dan en el orden del análisis de las construcciones sociales.

De esta forma, al momento de analizar la definición del género, "Esta se realiza en el momento que nace el bebé, a partir de la apariencia externa de los genitales" (Butler, 1996, p.113). Esta situación genera un nivel de complejidad para ser conceptualizado de forma genérica, ya que posee una influencia ejercida por las apreciaciones y construcciones epistemológicas, además de su influencia sobre la cultura y, en su defecto, sobre las sociedades, sobre las que finalmente, se ejercen las diferencias para construir el concepto.

La primera cuestión y dificultad que se plantea es ćcómo definir a la mujer? ¿En qué rasgos nos basamos que no sean los físicos? Este punto de partida ya complica el propio concepto de género, ya que la descripción de mujer en sí, sin hacerla en contrapunto de lo masculino es complicada, según el pensamiento occidental y la terminología lingüística. (Belmonte, 2008, p. 308)

Ahora, interesa extraer de lo antes dicho que las construcciones sociales que definen teóricamente el concepto de mujer están delimitadas por las diferentes maneras en que se ha definido la mujer y, en esencia, con el peso cultural que recae sobre su cuerpo; así, "La materialidad de los 
cuerpos que no solo tenemos, sino que somos, se construye como base de nuestras identidades, como nexo con lo inmaterial y como elemento de valoración moral” (Enguix y González, 2018, p. 4). Entonces, deben relacionarse el hecho de que las acciones no van solo contra el feminismo y el género, sino que se ejecutan sobre los cuerpos, los cuales tradicionalmente se han delimitado desde una visión de lo masculino. Venegas (2010):

Butler asegura que el feminismo es, por definición, una epistemología de y para el cambio social, que contiene en sí misma un potencial transformador. En este sentido, señala que entiende el género como norma (constrictivo, por tanto) y como experiencia (fenomenológicamente). (p. 150)

Estas digresiones, pueden determinar dicho concepto, pero sería estructurar un concepto poco acertado, ya que dicha orientación se ha permeado de la influencia que ha tenido la hegemonía masculina; sin embargo: "En cambio cuando se habla de mujer, se menciona como un conjunto de características diferentes, más concretas que la definición en sí de ser humano" (Belmonte, 2008, p. 308). Así que, la definición sobre la mujer ha estado mediada por lo teórico-conceptual al margen del hombre.

Pierre Bourdieu, plantea la dificultad que tiene poder analizar la perspectiva de género, él se basa en que el orden social masculino está tan arraigado en la sociedad que no hay que buscar explicación, y se plantea como la explicación natural, con lo cual complica sustancialmente el concepto de género desde un punto de vista neutro, sin la concepción masculina predominante. (Belmonte, 2008, p. 308)

Acontece, además, que la principal dificultad para definir el concepto de género y mujer es la fuerte influencia que ejerce la construcción social a partir del orden establecido por la hegemonía masculina. A partir de esta 
condición, se puede adherir la postura de la corriente del pensamiento posestructuralista, según la cual "se debe rechazar todo intento de definición de mujer, ya que cualquier apreciación es una forma de estereotipar y de encasillar a la mujer" (p.309). Pues bien, las feministas posestructuralistas plantean una nueva forma de construir el concepto de mujer desde un planteamiento distante del orden influenciado por la masculinidad, evocando de manera integral al ser humano:

La contribución distinguida de Simone de Beauvoir en la formulación, "uno no nace, sino que se convierte en mujer". De acuerdo con el marco anterior, el término 'mujer' designa un conjunto fijo e idéntico de hechos corporales naturales (una presunción, por el camino, que se ve seriamente desafiado por el continuo del cromo variaciones mosomales), y el término 'mujer' designa una variedad de modos a través de los cuales esos hechos adquieren significado cultural. (Butler, 1986, p. 35)

Sentadas las anteriores premisas, se constituyen en una acertada forma de acercarse al concepto y descripción teórica de la mujer. Por tanto, define elementos que permiten concluir con un acercamiento a una definición conceptual sobre dicha condición generalizada. El concepto de mujer emerge desde diferentes orientaciones y ejerce influencia directa sobre esta, con definiciones que se dan desde lo sociocultural, biológico, dogmático, religioso, entre otras formas que históricamente han influenciado su concepción por el orden impuesto por la masculinidad y que han tenido impacto sobre tal concepto de mujer.

Por su parte, el género al ser una construcción social se instala como un discurso que opera en función de definir lo que es o no legítimo para hombres y mujeres en el contexto de las conductas sexuadas, y es uno de los principios fundamentales con los que se estructura la sociedad en función de las formas de socialización, ya que "es una construcción simbólica, establecida sobre los datos biológicos de la diferencia sexual" 
(Lamas, 2003, p. 13). El género, entonces, es una "categoría que analiza cómo se definen, representan y simbolizan las diferencias sexuales en una determinada sociedad" (Inmujeres, 2007, p. 73). Ante las anteriores descripciones de los conceptos de violencia y género, se realiza un estudio que permite escudriñar en las diferentes conceptualizaciones sobre la violencia contra las mujeres. Hay que entender: "que la violencia contra las mujeres existe desde siempre y en el mundo entero con variantes de concienciación, sensibilización, datos estadísticos, leyes y/o políticas públicas con diferencias abismales entre países en su aplicación” (Álvarez, 2006, p, 46). Así es viable dimensionar que el fenómeno ha sido abordado desde varias perspectivas con diferentes matices y configuraciones que evidencian su interpretación en los diferentes contextos culturales y sociales donde se ha presentado la problemática.

\author{
Variabilidad y diversidad, tendencia a invisibilizar a la mujer, género \\ como enfoque relacional que no define per se, cambios por momentos de \\ avance o retroceso en el asunto, posiciones culturales influyentes, \\ influencia de traducciones literales, algunas aceptadas y otras \\ rechazadas. (Álvarez, 2006, p, 52)
}

Cabe analizar desde una postura crítica, las implicaciones que traen para la sociedad las acciones de violencia contra las mujeres; esto, de acuerdo con las evidencias plasmadas en los resultados de los estudios e investigaciones sobre el fenómeno y donde surgen los cuestionamientos ante la efectividad de los mecanismos de protección de las mujeres en el mundo y para lo cual hace un interesante análisis la investigadora cuando formula el interrogante. Según Álvarez (2006):

¿Qué incluiríamos siempre en una definición de una de las aristas a la problemática de nuestras mujeres y que incluya la perspectiva de género y los DH?: la connotación de proceso, el énfasis en lo cotidiano, el poder como fin, el cuido a la integridad de las personas, la agresión y el deseo 
de posesión del cuerpo del otro/a, la inclusión del contexto sociocultural, el impacto en la familia. (p. 52)

Ente interrogante debe ser analizado desde los cimientos de la sociedad colombiana y de sus instituciones; dentro de ellas, las de carácter castrense y militaristas, como son las Fuerzas Armadas y la Policía, donde las estructuras no están diseñadas ni pensadas desde una perspectiva de género, con condiciones incluyentes y sobre una base sólida por el respeto de los derechos humanos de las mujeres que las integran. Ante tal descripción, las "relaciones de poder en las que los valores masculinos son dominantes y universales [y así] la experiencia femenina es silenciada cuando se universaliza la experiencia humana en la del hombre" (Soto, 2013, p. 12). En este sentido, al profundizar en el concepto de patriarcado, es válido afirmar que el género es una construcción social y cultural, asociada a las relaciones de dominación que configuran la diferencia y las identidades que se fundamentan en las funciones biológicas sexuadas y definiendo lo que tradicionalmente se acepta en la sociedad patriarcal, apropiándola como conducta válida para hombres y mujeres.

Sin embargo, es significativo asumir que "El patriarcado es un concepto que acompaña desde el principio a las ciencias sociales. Ya en el siglo XX, Weber se refirió a este como uno de los subtipos de autoridad dentro de las formas de legitimidad tradicional" (Talego, 2012, p. 186). Lo cual considera que el concepto ha estado ligado a la evolución de la sociedad occidental, la cual cultural e históricamente lo ha avalado como forma legítima integrada; de ahí que la sociedad patriarcal esté definida por ser "aquel orden social genérico de poder, basado en un modo de dominación cuyo paradigma es el hombre" (Lagarde, 1996, p.52). Todas estas observaciones, facilitan asumir que dichas conductas se establecen como estereotipos en función de los cuales la sociedad define estructuralmente formas de pensar y actuar, configurando los sistemas de valores, ideologías 
y concepciones de mundo, pasando desapercibidas para la mayoría de las personas en la sociedad.

Así que el sistema de diferenciación "son sistemas binarios que oponen el hombre a la mujer, lo masculino a lo femenino y esto, no en un plan de igualdad sino en un orden jerárquico" (Conway, Bourque y Scott, 1996, p. 6). Estas diferencias sexuales, son espacios de relación en las que, desde la infancia, se configuran, se refuerzan y se producen dichas características; así que estos contextos de relación son instituciones sociales como la familia, la escuela, la religión, el ámbito laboral, entre otros, ya que, "El patriarcado, en tanto que institución, supone una violencia estructural que favorece relaciones desiguales e injustas en cuanto a recursos y medios, aspiraciones y oportunidades" (Izquierdo, citado en Talego, 2012, p. 195196). Se logra finalizar con que son acciones diferenciales que procuran invisibilizar a la mujer y muchas veces fomentan las formas de discriminación con configuraciones más radicales, instauradas como lógicas de violencia que poseen influencia directa sobre los sujetos, que en este caso son las mujeres.

\section{Efectos del poder en el dominio del cuerpo de la mujer y de la mujer policía}

Así, se implementan acciones que limitan la movilidad en algunos casos hasta la física impuesta sobre las mujeres, al ser sujetos regulados por la asignación de tareas; de ahí que, el cuerpo ha sido un "campo político; las relaciones de poder operan sobre él una presa inmediata; lo cercan, lo marcan, lo doman, lo someten a suplicio, lo fuerzan a unos trabajos, lo obligan a unas ceremonias, exigen de él unos signos" (Foucault, 1976, p.26). Ante este panorama, el cuerpo de la mujer ha estado en medio del control por el machismo en la sociedad colombiana, desplegando acciones de dominio en los diferentes contextos sociales, lo que orienta a moldear 
su actitud por medio de mecanismos desplegados por la política en su afán por dominarla.

Otro aspecto importante de carácter conceptual se relaciona con las tecnologías de poder, que facilitan el dominio sobre los sujetos, ya que "A estos métodos que permiten el control minucioso de las operaciones del cuerpo, que garantizan la sujeción constante de sus fuerzas y les imponen una relación de docilidad-utilidad, es a lo que se puede llamar las 'disciplinas"' (Foucault, 1976, p. 126). Esto se dimensiona, cuando Ferrero (2005) lo define como "el proceso de disolución de una conciencia femenina esclavizada por los mandatos de la sociedad patriarcal capitalista tardía” (p. 13). Así, emergen las acciones sobre la conciencia de la mujer al punto de buscar, de forma sistemática y producto de las prácticas del patriarcado, su esclavización como intencionalidad del capitalismo.

Simone de Beauvoir es muy clara en su análisis sobre la feminidad en las sociedades occidentales. Desde muy pequeñas, ya en sus experiencias fundantes, las niñas se ven expuestas a mensajes, relatos, mitos, documentos y otras formas de simbolización según las cuales la mujer es jerárquicamente inferiorizada frente al hombre. (Ferrero, 2005, p. 11)

Con esto, la implementación de la feminidad en las sociedades occidentales se ha llevado a cabo por medio de operaciones de adoctrinamiento y educación sobre la manera en que deben comportarse, por medio de dispositivos que regulan la conducta, donde se recalca de forma racional el rol que deben asumir en una sociedad dominada y controlada por el poder que ejercen los hombres; esto ha permitido que el machismo, el patriarcalismo y la misoginia, entre otras manifestaciones, hayan ido en contra de la integridad de las mujeres por medio de prácticas de violencia de género en las sociedades latinoamericanas y en la sociedad colombiana. Según Foucault (1976): 
$\mathrm{Y}$, sin embargo, del uno al otro, puntos de cruce. L'Homme-machine de La Mettrie es a la vez una reducción materialista del alma y una teoría general de la educación, en el centro de las cuales domina la noción de "docilidad" que une al cuerpo analizable el cuerpo manipulable. Es dócil un cuerpo que puede ser sometido, que puede ser utilizado, que puede ser trasformado y perfeccionado. Los famosos autómatas, por su parte, no eran únicamente una manera de ilustrar el organismo; eran también unos muñecos políticos, unos modelos reducidos de poder. (p.125)

Se puede añadir con el sano criterio que en la conceptualización foucaultiana se determina que la reducción del cuerpo y la trascendencia del alma se dan por medio de la dominación y de las prácticas de los hombres en el patriarcado, estableciendo escenarios de sometimiento, exclusión y adoctrinamiento sobre la mujer. Para concluir, muchos de estos hechos de violencia son orquestados por el poder de instituciones sociales, como la disciplina castrense, la religión, los cuarteles, la cárcel, la escuela, la familia, los gobiernos, entre otras instituciones y estructuras hegemónicas que someten su cuerpo, su voluntad y sus decisiones.

\section{Conclusiones}

Se pudo determinar a través de la aplicación de instrumentos, encuestas y entrevistas utilizadas en la investigación, la existencia de violencia de género dentro del centro de formación policial y que está ligada al tipo de violencia psicológica-emocional. Además, las participantes comprenden el fenómeno en la sociedad y, según el nivel de formación, comprenden la gravedad del fenómeno existente. El personal de mujeres estudiantes de policía dimensiona el significado de la violencia, identifican los tipos de violencia de género contra las mujeres y señalaron que el más relevante de los tipos es el psicológico-emocional. 
Se logró mostrar por medio del análisis cuantitativo y cualitativo de los instrumentos aplicados que se presenta violencia de género en algunos ámbitos de la institución, condición heredada de la formación y estructura machista, castrense y militarista como escenario laboral general más relevante y que deja en evidencia que, en algunos casos, las mujeres estudiantes de policía no presentan todas las condiciones para su desempeño en la institución. A su vez, llaman la atención las acciones ejercidas por la comunidad, ya que también se ejercen manifestaciones de violencia de género con ellas.

Ante las manifestaciones de la problemática y su comprensión como delito, se concluyó en el estudio que el mecanismo al que recurren las mujeres estudiantes de policía para hacer frente a los actos de violencia es la denuncia, y como mecanismo para la solución de la problemática buscan ayuda en la institución. Por tal motivo, deben generarse acciones para el fortalecimiento de la denuncia, reestructurar dependencias disciplinarias, ser más efectivas y darles un tratamiento inmediato a los actos de violencia en la institución.

En cuanto al ocultamiento del fenómeno de la violencia de género, se logró concluir que fueron señaladas las mismas mujeres como las funcionarias que más desarrollan dichas acciones violentas; así que es necesario liderar estrategias de divulgación con bases éticas que permitan comprender la dimensión del fenómeno en el interior del centro de formación policial, generando mecanismos en materia de orientación y sensibilización para las mujeres integrantes de la institución.

La institución debe brindarles condiciones integrales de equidad, igualdad, dignidad humana y respeto por su condición de género; para lo cual la institución debe mantener acciones encaminadas, desde la pedagogía y la prevención, a concientizar al personal que conforma la institución policial sobre la importancia del respeto de los derechos y libertades y sobre las 
implicaciones disciplinarias, judiciales y penales que poseen los actos de este fenómeno de género en contra de las mujeres en el país.

Finalmente, en materia de abusos de autoridad, machismo, misoginia y discriminación, entre otras formas de violencia de género contra la mujer, la institución debe velar por mantener sus condiciones integrales y por ejercer un control permanente a las órdenes impartidas en las que se comprometa el personal femenino de la institución; esto con el fin de evitar abusos de autoridad o actos que lesionen los derechos humanos de las mujeres estudiantes de policía en el centro educativo policial y en la institución.

\section{Referencias}

Abreu, J. (2012). Hipótesis, método y diseño de investigación (hypothesis, method y research design). Daena: International Journal of Good Conscience, 7(2), 187-197. http://www.spentamexico.org/v7-n2/7(2)187-197.pdf

Álvarez, O. (2006). El enfoque de género y la violencia contra las mujeres: aproximación al análisis de los conceptos. Revista venezolana de estudios de la mujer, 11, (26). http://ve.scielo.org/scielo.php?pid=S1316$37012006000100003 \&$ script $=$ sci arttext

Amigot, P. y Pujal, M. (2009). Una lectura del género como dispositivo de poder. Sociológica México, 24(70), 115-151. http://www.scielo.org.mx/pdf/soc/v24n70/v24n70a5.pdf

Belmonte, R. (2008). Concepto de género: Reflexiones. Ensayos: Revista de la Facultad de Educación de Albacete, (23), 307-314.

Bobea, L. (2008). Mujeres en uniforme: la feminización de las fuerzas armadas. Un estudio del caso dominicano. Nueva Sociedad, (213), 64-79. http://www.plataformademocratica.org/Publicacoes/Publicacao 8624 em 310 5_2011_10_51_46.pdf

Bourdieu, P. (1998). La dominación masculina (7. ${ }^{\mathrm{a}}$ ed.). Anagrama, S. A. 
Butler, J. (1986). Sex and gender in Simone de Beauvoir's Second Sex. Yale French Studies, (72), 35-49.

http://blogs.law.columbia.edu/critique1313/files/2019/10/Butler-on- Beauvoir.pdf

Butler, J. (1996). Variaciones sobre sexo y género: Beauvoir, Wittig y Foucault. En: M.

Lamas (compiladora). El género: La construcción cultural de la diferencia sexual, (pp. 291-312) Bonilla Artigas Editores.

Castrillón, L. y Von Chrismar, P. (2013). Mujer y fuerzas armadas en el contexto sudamericano: una visión desde Chile. Estudios de Seguridad y Defensa, (2), 91-116. http://esd.anepe.cl/wp-content/uploads/2014/04/art4.pdf

Cherryholmes, H. y Pomares, M. (1999). Poder y crítica: Investigaciones postestructurales en educación. http://www.sidalc.net/cgi$\underline{\text { bin/wxis.exe/?IsisScript=AGRIUAN.xis\&method=post\&formato }=2 \& \text { cantidad }=1 \& \mathrm{e}}$ xpresion $=m f n=019203$

Conway, J., Bourque, S. y Scott, J. (2000). El concepto de género. En: M. Lamas (compiladora). El género: La construcción cultural de la diferencia sexual (pp. 41-52). Bonilla Artiga Editores.

Delgado, C., Sánchez, C. y Fernández, A. (2012). Atributos y estereotipos de género asociados al ciclo de la violencia contra la mujer. Universitas Psychologica, 11, (3), 769- 777 . http://revistas.javeriana.edu.co/index.php/revPsycho/article/view/769/287

Derdoy, M. (2013). Incorporación e integración de las mujeres en las Fuerzas Armadas. Revista Conjeturas Sociológicas, 80-91. http://csociales.fmoues.edu.sv/revista/files/art5.pdf

Enguix B., y González M. (2018). Cuerpos, mujeres y narrativas: Imaginando corporalidades y géneros. Athenea digital: revista de pensamiento e investigación social, 18(2), 003 . https://ddd.uab.cat/pub/athdig/athdig a2018v18n2/athdig a2018v18n2e1956.pd $\underline{\mathrm{f}}$

Espinar, E., y Mateo, M. (2007). Violencia de género: reflexiones conceptuales, derivaciones prácticas. Papers: Revista de Sociología, (86), pp. 189-201 
Estrada, Á. M. (1997). Los estudios de género en Colombia: entre los límites y las posibilidades. Nómadas, (6). https://www.redalyc.org/pdf/1051/105118999004.pdf

Facio, A. (2003). Asegurando el futuro. Las instituciones nacionales de derechos humanos y los derechos reproductivos. Fondo de Población de las Naciones Unidas.

Ferrero, A. M. (2005). De la teoría por otros medios: Simone de Beauvoir y sus ficciones. Revista Clepsydra: revista de estudios de género y teoría feminista, 4, 9-21. http://www.bdigital.unal.edu.co/50624/1/delateoriaporotros.pdf

Foucault, M. (1976). Historia de la Sexualidad I. La Voluntad de Saber. Siglo XXI Editores.

Foucault, M. (2002). Vigilar y Castigar. Nacimiento de la Prisión. Siglo XXI Editores.

Foucault, M. (2008). Tecnologías del yo. Yotros textos a fines. Paidós Ibérica Ediciones SA.

Gómez, C., Murad. R. y Calderón, M. (2013). Historias de violencia, roles, prácticas y discursos legitimadores. Violencia contra las mujeres en Colombia 2000 - 2010. Ministerio de Salud.1-95.

https://www.minsalud.gov.co/sites/rid/Lists/BibliotecaDigital/RIDE/INEC/INV/ 7\%20-\%20VIOLENCIA\%20CONTRA\%2OLAS\%20MUJERES\%20EN\%20COLOMB IA.pdf

Hernández, R., Fernández, C. y Lucio, M. (6ta Ed.) (2014). Metodología de la investigación. McGraw-Hill. http://observatorio.epacartagena.gov.co/wpcontent/uploads/2017/08/metodologia-de-la-investigacion-sextaedicion.compressed.pdf

Instituto Nacional de las Mujeres [Inmujeres]. (2007). Glosario de género. http://cedoc.inmujeres.gob.mx/documentos download/100904.pdf

Lagarde, M. (1996). Género y feminismo: Desarrollo humano y democracia. Horas y horas.

Lamas, M. (2003). El género: La construcción cultural de la diferencia sexual. Grupo Editorial Miguel Ángel Porrúa. https://www.semanticscholar.org/paper/Elg\%C3\%Agnero\%3A-la-construcci\%C3\%B3n-cultural-de-la-sexualLamas/54bbcbccf52904005a53bda34f673booed364898?p2df 
Lucero, M. (26-28 de noviembre de 2008). Fuerzas armadas sudamericanas en el contexto global. La situación de las mujeres dentro del ámbito de la defensa y la seguridad. IV Congreso de Relaciones Internacionales. La Plata. Argentina: http://sedici.unlp.edu.ar/bitstream/handle/10915/40854/Documento completo.p df? sequence $=1$

Organización Mundial de la Salud [OMS]. (2013). Violencia contra la mujer. Violencia de pareja y violencia sexual contra la mujer.

http://www.who.int/mediacentre/factsheets/fs239/es/

Pérez del Rio, T. (s. F.). La violencia de género en el trabajo: el acoso sexual y el acoso moral por razón de género. Temas Laborales, 91, 175-203. https://dialnet.unirioja.es/descarga/articulo/2481175.pdf

Policía Nacional de Colombia. (2010). Capítulo V: Satisfacción del talento humano para hacer bien lo que nos gusta y sentirnos bien con nosotros mismos. En:

Lineamiento de política 1 Direccionamiento policial basado en el humanismo con responsabilidad. Imprenta Nacional de Colombia.

Presidencia de la República de Colombia. (1991). Constitución Política de Colombia. http://www.secretariasenado.gov.co/index.php/constitucion-politica

Puyana, V. Y. (2004). Los Estudios de mujer y género en la Universidad Nacional de Colombia. Ponencia Seminario «Género, Mujeres y Saberes en América Latina. Entre el Movimiento Social, la Academia y el Estado». https://www.researchgate.net/profile/Montserrat Sagot/publication/30757549 Genero mujer es_y_saberes en_America_Latina/links/02e7e51d1ac3co8099000000/Generomujeres-y- saberes-en-America-Latina.pdf

Rebolledo, L. (2001). Impacto de los estudios de género en la democratización de las instituciones de educación superior. La ventana. Revista de Estudios de Género, 2(13), 75-93.

http://148.202.18.157/sitios/publicacionesite/pperiod/laventan/Ventana13/venta na13-3.pdf

Ruiz, R. C. (2013). La igualdad de género en las fuerzas armadas desde una perspectiva constitucional. Instituto Español de Estudios Estratégicos. (121). www.ieee.es/Galerias/fichero/docs_opinion/2013/DIEEEO1212013 IgualdadGeneroFFAA CRuiz-Rico.pdf 
Soto, G. (2013). El Estado como garante de los derechos fundamentales de las mujeres en Venezuela bajo el marco de la Nueva Ley Orgánica sobre el derecho de las mujeres a una vida libre de violencia. [Tesis Doctoral Universidad Nacional de Educación a Distancia]. http://62.204.194.45/fez/eserv/tesisuned:DerechoGsoto/Documento.pdf

Talego, F., Florido del Corral, D. y Cantó, S. (2012). Reconsiderando la violencia machista. Patriarcado, relaciones de pareja y sadismo. Revista Andaluza de Antropología, 3, 2174-6796. https://idus.us.es/bitstream/handle/11441/25969/file_1.pdf?sequence=1

Valencia, L. P. (2014). Retos del manejo de información sobre violencia basada en género (VBG) en el marco del conflicto armado. El caso de Medellín. Opinión Jurídica, (13), 26, 49-68. http://revistas.udem.edu.co/index.php/opinion/article/view/955/959

Venegas, M. (2010). La maldición de ser niña: Estructuralismo, postestructuralismo y teoría de la práctica en género y sexualidad. Papers: revista de Sociología, 95(1), 139-156.

https://ddd.uab.cat/pub/papers/o2102862v95n1/02102862v95n1p139.pdf

Villalobos, P. (2010). Participación de las mujeres en las fuerzas armadas y de orden: Políticas para promover la inserción de la mujer en la vida pública: mesa de trabajo pro-género. Memoria chilena. 1-25. http://www.memoriachilena.cl/602/w3article-125917.html

Zubieta, E., Beramendi, M., Sosa, F. y Torres, J. (2011). Sexismo ambivalente, estereotipos y valores en el ámbito militar. Revista de Psicología (PUCP), 29(1), 101-130. http://www.scielo.org.pe/pdf/psico/v29n1/ao4v29n1.pdf 